\title{
Necessary and Sufficient Conditions for Inner Functions to Be in $Q_{K}(p, p-2)$-Spaces
}

\begin{abstract}
Atte Reijonen
Department of Physics and Mathematics, University of Eastern Finland, P.O. Box 111, 80101 Joensuu, Finland

Correspondence should be addressed to Atte Reijonen; atte.reijonen@uef.fi
\end{abstract}

Received 17 July 2015; Accepted 8 November 2015

Academic Editor: José Á Peláez

Copyright (C) 2015 Atte Reijonen. This is an open access article distributed under the Creative Commons Attribution License, which permits unrestricted use, distribution, and reproduction in any medium, provided the original work is properly cited.

Under some regularity conditions on $K$, inner functions in $Q_{K}(p, p-2)$-spaces are characterized in the following way: an inner function belongs to $Q_{K}(p, p-2)$ if and only if it is a Blaschke product associated with $\left\{z_{n}\right\}$ satisfying $\sup _{a \in \mathbb{D}} \sum_{n} K\left(1-\left|\varphi_{a}\left(z_{n}\right)\right|\right)<\infty$, where $\varphi_{a}(z)=(a-z) /(1-\bar{a} z)$. The result generalizes earlier theorems in (Essén et al., 2006) and (Pérez-González and Rättyä, 2009).

\section{Introduction}

Inner functions are bounded analytic functions in the unit $\operatorname{disc} \mathbb{D}=\{z \in \mathbb{C}:|z|<1\}$ such that their moduli are one almost everywhere on the boundary $\mathbb{T}=\{z \in \mathbb{C}:|z|=$ $1\}$. Each inner function can be represented as a product of a Blaschke product and a singular inner function [1]. Singular inner functions are of the form

$$
S(z)=\exp \left(-\int_{\mathbb{T}} \frac{z+w}{z-w} d \sigma(w)\right), \quad z \in \mathbb{D},
$$

where $\sigma$ is a positive measure on $\mathbb{T}$ which is singular with respect to the Lebesgue measure, and, for $w \in \mathbb{T}$ and $m \in$ $\mathbb{N} \cup\{0\}$,

$$
B(z)=w z^{m} \prod_{n} \frac{\left|z_{n}\right|}{z_{n}} \frac{z_{n}-z}{1-\bar{z}_{n} z}, \quad z \in \mathbb{D},
$$

is a Blaschke product associated with $\left\{z_{n}\right\} \subset \mathbb{D} \backslash\{0\}$ if $\sum_{n}\left(1-\left|z_{n}\right|\right)<\infty$ [2]. Information about inner functions can be found in [3]; see also [4-6], for example.

An analytic function $f$ in $\mathbb{D}$ belongs to $Q_{K}(p, q)$ for $p \in$ $(0, \infty)$ and $q \in(-2, \infty)$ if

$$
\sup _{a \in \mathbb{D}} \int_{\mathbb{D}}\left|f^{\prime}(z)\right|^{p}(1-|z|)^{q} K(g(z, a)) d A(z)<\infty .
$$

Here $K:[0, \infty) \rightarrow[0, \infty)$ is such that $K(1)>0, g(z, a)=$ $\log |(1-\bar{a} z) /(a-z)|$ is Green's function, and $d A(z)$ is the
Lebesgue area measure. Information about $Q_{K}(p, q)$-spaces can be found in $[7,8]$; see also the beginning of the next section.

Our purpose is to study the behaviour of inner functions in $Q_{K}(p, q)$-spaces. In particular, we are interested in the Möbius invariant $Q_{K}(p, p-2)$-spaces. Related to $Q_{K}(p, p-2)$, it is proved that only inner functions there are Blaschke products if $K$ satisfies certain regularity conditions; see Proposition 7. Under corresponding assumptions, our main result, Theorem 1, gives a complete characterization of inner functions in the spaces. The results generalize [9, Theorems 1.3 and 1.4] and the essential content of [10, Theorem 5.1].

Theorem 1. Let $p \in(1 / 2, \infty)$, and assume that there exists $\alpha, \beta \in(1-\min \{p, 1\}, \min \{p, 1\})$ such that a nondecreasing $K$ satisfies $r^{-\alpha} K(r) \searrow$ and $r^{-\beta} K(r)>$ for $r \in(0,1)$. Then an inner function belongs to $Q_{K}(p, p-2)$ if and only if it is a Blaschke product associated with a sequence $\left\{z_{n}\right\}$ satisfying

$$
\sup _{a \in \mathbb{D}} \sum_{n} K\left(1-\left|\varphi_{a}\left(z_{n}\right)\right|\right)<\infty,
$$

where $\varphi_{a}(z)=(a-z) /(1-\bar{a} z)$.

Note that an assertion similar to Theorem 1 can be found in [11]. The proof there, however, contains some inaccuracies and does not seem to yield the claimed result. It is also worth 
noticing that the weight function $K$ is essentially so-called normal one in Theorem 1 [12].

The notation $f \nearrow$ means that $f$ is essentially increasing; that is, $f\left(r_{1}\right) \lesssim f\left(r_{2}\right)$ for $r_{1} \leq r_{2}$. The term essentially decreasing, shortly $f \searrow$, is understood in an analogous manner. Here $a \asymp b$ if $a \leqslant b$ and $a \gtrsim b$. The notation $a \leqslant b$ is used if there exists a constant $C>0$ such that $a \leq C b$, and $a \gtrsim b$ is understood in a similar manner. Note that the constant $C$ may depend on fixed parameters or functions.

The remainder of the paper is organized as follows. In the next section, some auxiliary results are presented. Section 3 contains necessary conditions for singular inner functions to be in $Q_{K}(p, q)$-spaces and the main purpose of the last section is to prove Theorem 1. Also Proposition 7 and an alternative version of Theorem 1 are stated and proved in the last section.

\section{Auxiliary Results}

In this section, we present auxiliary results related to $Q_{K}(p, q)$-spaces and inner functions. The weight function $K$ plays a crucial role in these results.

For simplicity, we denote the following conditions:

(a) $K$ is continuous and nondecreasing.

(b) $\int_{0}^{1}(1-r)^{q} K(\log (1 / r)) r d r<\infty$.

(c) $K(r) \asymp K(2 r)$ for $r \in(0, \infty)$.

If (b) does not hold, then $Q_{K}(p, q)$ contains constant functions only [7]. Hence it is natural to assume (b), even though it would not be necessary. Nevertheless, it will always be mentioned if an assumption is made.

In the next lemma, we recall some basic properties of $\mathrm{Q}_{K}(p, q)$-spaces. It follows by combining results of [7]. For the lemma, we denote that $f \in \mathscr{H}(\mathbb{D})$ belongs to $\mathscr{B}^{\alpha}$ for $\alpha \in(0, \infty)$ if $\sup _{z \in \mathbb{D}}\left|f^{\prime}(z)\right|(1-|z|)^{\alpha}<\infty$. If $\alpha=1$, then we use the notation $\mathscr{B}$. Here $f \in \mathscr{H}(\mathbb{D})$ means that $f$ is analytic in $\mathbb{D}$. Moreover, write $A_{q}^{p}=Q_{K}(p, q)$ if $K \equiv 1$.

Lemma A. Let $p \in(0, \infty)$ and $q \in(-2, \infty)$, and assume that $K$ satisfies ( $a)$ and (b). Then the following statements are valid:

(i) $Q_{K}(p, q) \subset \mathscr{B}^{(q+2) / p}$. Moreover, $Q_{K}(p, q)=\mathscr{B}^{(q+2) / p}$ if and only if

$$
\int_{0}^{1}(1-r)^{-2} K\left(\log \frac{1}{r}\right) r d r<\infty .
$$

(ii) If $K_{1}(r)=K(r)$ for $r \in(0,1)$ and $K_{1}(s)=K(1)$ for $s \in[1, \infty)$, then $Q_{K_{1}}(p, q)=Q_{K}(p, q)$.

(iii) $f \in Q_{K}(p, q)$ if and only if $f \in \mathscr{H}(\mathbb{D})$ and

$\sup _{a \in \mathbb{D}} \int_{\mathbb{D}}\left|f^{\prime}(z)\right|^{p}(1-|z|)^{q} K\left(1-\left|\varphi_{a}(z)\right|\right) d A(z)<\infty$.

(iv) $A_{q}^{p} \subset Q_{K}(p, q)$. Moreover, $A_{q}^{p}=Q_{K}(p, q)$ if and only if $K(0)>0$.
It is worth noticing that the statements (i)-(iii) of Lemma $\mathrm{A}$ are valid even if $K$ is discontinuous. This can be seen by looking the proofs of [7, Theorems 2.1 and 3.1].

Denote that $Q(I)=\{r \xi: 1-|I|<r<1, \xi \in I\}$, where $I$ is a subarc of $\mathbb{T}$ such that $|I|<1$. Then a positive measure $\mu$ on $\mathbb{D}$ is a $K$-Carleson measure if

$$
\sup _{I} \int_{Q(I)} K\left(\frac{1-|z|}{|I|}\right) d \mu(z)<\infty
$$

Now we can characterize $Q_{K}(p, q)$-spaces by using $K$ Carleson measures. This result has been earlier presented in [11, Theorem 1], but practically, it follows by modifying the proof of [10, Theorem 3.1].

Lemma B. Let $p \in(0, \infty)$ and $q \in(-2, \infty)$, and assume that $K$ satisfies (a)-(c) and

$$
\int_{0}^{1} \varphi_{K}(r) \frac{d r}{r}<\infty, \text { where } \varphi_{K}(r)=\sup _{t \in(0,1)} \frac{K(r t)}{K(t)} .
$$

Then $f \in Q_{K}(p, q)$ if and only if $f \in \mathscr{H}(\mathbb{D})$ and $\left|f^{\prime}(z)\right|^{p}(1-$ $|z|)^{q} d A(z)$ is a K-Carleson measure.

The next lemma shows that under certain conditions one may apply the Schwarz-Pick theorem without any essential loss of information. Before the lemma, we underline that the assumptions (a)-(c) are not necessary therein. In fact, it suffices to assume that $K:(0,1) \rightarrow[0, \infty)$ but, of course, if (b) does not hold, then the statements of the lemma are trivial.

Lemma 2. Let $p \in[1, \infty)$ and $q \in(-2, \infty)$, and let $\phi$ be an inner function. If

(i) $p=1$ and

$$
\begin{aligned}
& \underset{r \in(\delta, 1)}{\operatorname{ess} \sup }(1-r)^{-q} K\left(\frac{1-r}{1-\delta}\right)^{-1} \\
& \cdot \int_{\delta}^{r}(1-s)^{q-1} K\left(\frac{1-s}{1-\delta}\right) d s \leqslant 1, \quad \delta \in(0,1) ;
\end{aligned}
$$

(ii) $p \in(1, \infty)$ and

$$
\begin{aligned}
& \sup _{r \in(\delta, 1)} \int_{\delta}^{r}(1-s)^{q-p} K\left(\frac{1-s}{1-\delta}\right) d s \\
& \cdot\left(\int_{r}^{1}(1-s)^{q /(1-p)} K\left(\frac{1-s}{1-\delta}\right)^{1 /(1-p)} d s\right)^{p-1} \leq 1,
\end{aligned}
$$

$\delta \in(0,1)$

then

$$
\begin{gathered}
\int_{\delta}^{1}\left(1-\left|\phi\left(r e^{i \theta}\right)\right|\right)^{p}(1-r)^{q-p} K\left(\frac{1-r}{1-\delta}\right) d r \\
\lesssim \int_{\delta}^{1}\left|\phi^{\prime}\left(r e^{i \theta}\right)\right|^{p}(1-r)^{q} K\left(\frac{1-r}{1-\delta}\right) d r
\end{gathered}
$$

is satisfied for all $\delta \in(0,1)$ and almost all $\theta \in[0,2 \pi)$. 
Proof. Assume first that (i) holds, and denote $\omega_{\delta, q}(r)=(1-$ $r)^{q} K((1-r) /(1-\delta))$. Since $1-\left|\phi\left(r e^{i \theta}\right)\right| \leq \int_{r}^{1}\left|\phi^{\prime}\left(s e^{i \theta}\right)\right| d s$ for almost all $\theta \in[0,2 \pi)$, by Fubini's theorem and (i), we obtain

$$
\begin{aligned}
& \int_{\delta}^{1}\left(1-\left|\phi\left(r e^{i \theta}\right)\right|\right) \frac{\omega_{\delta, q}(r)}{1-r} d r \\
& \leq \int_{\delta}^{1} \int_{r}^{1}\left|\phi^{\prime}\left(s e^{i \theta}\right)\right| d s \frac{\omega_{\delta, q}(r)}{1-r} d r \\
& \quad=\int_{\delta}^{1}\left|\phi^{\prime}\left(s e^{i \theta}\right)\right| \int_{\delta}^{s} \frac{\omega_{\delta, q}(r)}{1-r} d r \\
& \quad \leq \int_{\delta}^{1}\left|\phi^{\prime}\left(s e^{i \theta}\right)\right| \omega_{\delta, q}(s) d s
\end{aligned}
$$

for almost all $\theta \in[0,2 \pi)$. If (ii) holds, then an application of [13, Theorem 2] yields

$$
\begin{aligned}
& \int_{\delta}^{1}\left(1-\left|\phi\left(r e^{i \theta}\right)\right|\right)^{p} \frac{\omega_{\delta, q}(r)}{(1-r)^{p}} d r \\
& \quad \leq \int_{\delta}^{1}\left(\frac{\omega_{\delta, q}(r)^{1 / p}}{1-r}\right)^{p}\left(\int_{r}^{1}\left|\phi^{\prime}\left(s e^{i \theta}\right)\right| d s\right)^{p} d r \\
& \quad \leq \int_{\delta}^{1}\left|\phi^{\prime}\left(r e^{i \theta}\right)\right|^{p} \omega_{\delta, q}(r) d r
\end{aligned}
$$

for almost all $\theta \in[0,2 \pi)$. This completes the proof.

Corollary 3. Let $p \in[1, \infty), q \in(-2, \infty)$, and $\alpha \in[0, \infty)$ such that $p>q+\alpha+1$, and let $\phi$ be an inner function. If $K$ satisfies $r^{-\alpha} K(r) \searrow$ for $r \in(0,1)$, then (11) holds for all $\delta \in$ $(0,1)$ and almost all $\theta \in[0,2 \pi)$.

Proof. By the assumption, we have

$$
\begin{aligned}
\int_{\delta}^{r} & (1-s)^{q-p} K\left(\frac{1-s}{1-\delta}\right) d s \\
\quad & \int_{\delta}^{r}(1-s)^{q-p} \frac{K((1-s) /(1-\delta))}{((1-s) /(1-\delta))^{\alpha}}\left(\frac{1-s}{1-\delta}\right)^{\alpha} d s \\
& \lesssim \frac{K((1-r) /(1-\delta))}{(1-r)^{\alpha}} \int_{0}^{r}(1-s)^{\alpha+q-p} d s \\
& =(1-r)^{q+1-p} K\left(\frac{1-r}{1-\delta}\right), \quad r \in(\delta, 1) .
\end{aligned}
$$

Similarly, for $p>1$, we obtain

$$
\begin{aligned}
& \left(\int_{r}^{1}(1-s)^{q /(1-p)} K\left(\frac{1-s}{1-\delta}\right)^{1 /(1-p)} d s\right)^{p-1} \\
& \quad \lesssim(1-r)^{p-q-1} K\left(\frac{1-r}{1-\delta}\right)^{-1}, \quad r \in(\delta, 1) .
\end{aligned}
$$

Now the assertion follows from Lemma 2 applying the formulas above.

It is worth noticing that [9, Lemma 2.1] is a special case of Lemma 2 . This is easy to see by choosing $K \equiv 1$ and $\alpha=0$ in Corollary 3.
Denote that $K$ satisfies (A), if (8) and (a)-(c) are satisfied, and (B) if (9) holds for $p \in(0,1]$ and (10) holds for $p \in(1, \infty)$. Related to (A) and (B), we end this section by proving the following consequence of Lemma 2.

Corollary 4. Let $p \in[1, \infty)$ and $q \in(-2, \infty)$, and assume that $K$ satisfies $(A)$ and $(B)$. Let $\phi=\prod_{n=1}^{k} \phi_{n}$, where $\phi_{n}$ is an inner function for all $n=1, \ldots, k$. Then $\phi \in Q_{K}(p, q)$ if and only if $\phi_{n} \in Q_{K}(p, q)$ for all $n=1, \ldots, k$.

Proof. Hölder's inequality yields $\left|\phi^{\prime}(z)\right|^{p} \leq$ $k^{p-1} \sum_{n=1}^{k}\left|\phi_{n}^{\prime}(z)\right|^{p}$. Hence $\phi \in Q_{K}(p, q)$ if $\phi_{n} \in Q_{K}(p, q)$ for all $n=1, \ldots, k$. The other implication follows by applying Lemmas B and 2 together with the fact that $|\phi| \leq\left|\phi_{n}\right|$ for all $n=1, \ldots, k$.

\section{Singular Inner Functions and $Q_{K}(p, q)$-Spaces}

This section contains necessary conditions for singular inner functions to be in $Q_{K}(p, q)$-spaces.

Write $I_{\xi}=I_{\xi}(|I|)=\left\{e^{i \theta}: \theta \in(\xi-|I| / 2, \xi+|I| / 2)\right\}$, where $\xi \in[0,2 \pi)$ and $|I| \in(0,1)$.

Lemma 5. Let $p \in[1, \infty)$ and $q \in(-2, \infty)$, and assume that $K$ satisfies $(A)$ and $(B)$. If $S$ is the singular inner function associated with a measure $\sigma$ and there exists $\xi \in[0,2 \pi)$ such that $\lim _{|I| \rightarrow 0^{+}} \sigma\left(I_{\xi}\right) /|I|=\infty$ and either

$$
\lim _{|I| \rightarrow 0^{+}}|I|^{q+2-p} \int_{|I| / \sigma\left(I_{\xi}\right)}^{1} r^{q-p} K(r) d r=\infty
$$

or

$$
\lim _{|I| \rightarrow 0^{+}}|I|^{q+2-p}\left(\frac{\sigma\left(I_{\xi}\right)}{|I|}\right)^{p} \int_{0}^{|I| / \sigma\left(I_{\xi}\right)} r^{q} K(r) d r=\infty
$$

then $S \notin Q_{K}(p, q)$.

Proof. Since $\lim _{|I| \rightarrow 0^{+}} \sigma\left(I_{\xi}\right) /|I|=\infty$, we may assume that $|I| / \sigma\left(I_{\xi}\right)<1$. Then, for $\theta \in(\xi-(|I| / 2), \xi+(|I| / 2))$, Lemma 2 yields

$$
\begin{aligned}
& \int_{1-|I|}^{1}\left|S^{\prime}\left(r e^{i \theta}\right)\right|^{p}(1-r)^{q} K\left(\frac{1-r}{|I|}\right) r d r \\
& \quad=\int_{1-|I|}^{1}\left(1-\left|S\left(r e^{i \theta}\right)\right|\right)^{p}(1-r)^{q-p} K\left(\frac{1-r}{|I|}\right) d r \\
& \quad \gtrsim \int_{1-|I|}^{1}\left(1-\exp \left(-\int_{\theta-|I|}^{\theta+|I|} \frac{1-r}{\left|r e^{i \theta}-e^{i t}\right|^{2}} d \sigma(t)\right)\right)^{p} \\
& \cdot(1-r)^{q-p} K\left(\frac{1-r}{|I|}\right) d r
\end{aligned}
$$




$$
\begin{aligned}
& \gtrsim \int_{1-|I|}^{1}\left(1-\exp \left(-\frac{\sigma\left(I_{\xi}\right)(1-r)}{|I|^{2}}\right)\right)^{p}(1-r)^{q-p} \\
& \cdot K\left(\frac{1-r}{|I|}\right) d r=|I|^{q+1-p} \\
& \cdot \int_{0}^{1}\left(1-\exp \left(-\frac{\sigma\left(I_{\xi}\right) s}{|I|}\right)\right)^{p} s^{q-p} K(s) d s \\
& \asymp|I|^{q+1-p} \int_{0}^{|I| / \sigma\left(I_{\xi}\right)}\left(1-\exp \left(-\frac{\sigma\left(I_{\xi}\right) s}{|I|}\right)\right)^{p} \\
& \cdot s^{q-p} K(s) d s+|I|^{q+1-p} \int_{|I| / \sigma\left(I_{\xi}\right)}^{1} s^{q-p} K(s) d s \\
& =: J_{1}\left(I_{\xi}\right)+J_{2}\left(I_{\xi}\right) .
\end{aligned}
$$

Since $1-e^{-x} \asymp x$ for all $x \in(0, M)$ with fixed $M \in(0, \infty)$, we obtain

$$
J_{1}\left(I_{\xi}\right) \asymp|I|^{q+1-p}\left(\frac{\sigma\left(I_{\xi}\right)}{|I|}\right)^{p} \int_{0}^{|I| / \sigma\left(I_{\xi}\right)} s^{q} K(s) d s .
$$

Hence the assertion follows by Lemma B.

If $\sigma\left(I_{\xi}\right)>0$ for all $|I|>0$, then $\lim _{|I| \rightarrow 0^{+}} \sigma\left(I_{\xi}\right) /|I|=\infty$ by [14, Theorem 7.15]. In particular, there exists always $\xi \in$ $[0,2 \pi)$ such that $\lim _{|I| \rightarrow 0^{+}} \sigma\left(I_{\xi}\right) /|I|=\infty$. Hence the following result is a direct consequence of Lemma 5.

Corollary 6. Let $p \in[1, \infty)$ and $q \in(-2, \infty)$, and assume that $K$ satisfies $(A)$ and (B). If $S$ is the singular inner function associated with a measure $\sigma$ and

$$
\begin{aligned}
\sup _{\xi \in[0,2 \pi)}|I|^{q+2-p} \int_{|I| / \sigma\left(I_{\xi}\right)}^{1} r^{q-p} K(r) d r=\infty, & \\
& |I| \longrightarrow 0^{+},
\end{aligned}
$$

then $S \notin Q_{K}(p, q)$.

\section{Blaschke Products and $Q_{K}(p, p-2)$-Spaces}

The main purpose of this section is to prove Theorem 1 . We begin by showing that all inner functions in $Q_{K}(p, p-2)$ are Blaschke products if $K$ satisfies certain regularity conditions. After that an alternative version of Theorem 1 is stated and proved; and finally, Theorem 1 follows by applying this result.

Proposition 7. Let $p \in(0, \infty)$ and $q \in(-2, \infty)$, and assume that a nondecreasing $K$ satisfies (b). Then the following statements are valid:

(i) If $p>q+2$, then only inner functions in $Q_{K}(p, q)$ are finite Blaschke products.

(ii) If $\int_{0}^{1} \varphi_{K}(r)(d r / r)<\infty$ and $\int_{1}^{\infty} \varphi_{K}(r)\left(d r / r^{2}\right)<\infty$, then only inner functions in $Q_{K}(p, p-2)$ are Blaschke products.
Proof. Since only inner functions in VMOA are finite Blaschke products [15], the statement (i) follows from the inclusions $Q_{K}(p, q) \subset \mathscr{B}^{(q+2) / p} \subset \mathrm{VMOA}$ for $p>q+2$.

By using [16, Lemma 2] twice together with [16, Corollary 3], [10, Lemma 2.3], and Lemma A(ii), we can assume that $K$ is second differentiable, $K(r) \asymp K(2 r)$ for $r \in(0, \infty), K(s) / s \rightarrow \infty$ as $s \rightarrow 0^{+}$, and $K(t)=K(1)$ for $t \in[1, \infty)$. Hence it is clear that (A) holds. Moreover, the condition $\int_{0}^{1} r^{-2} K(r) d r=\infty$ follows from [11, Lemma 3] and (B) with $q=p-2$ follows from the proof of Corollary 3 using [10, Lemma 2.2].

We may assume that $p \in[1, \infty)$ because the inclusion $Q_{K}(p, p-2) \subset \mathscr{B}$ yields $Q_{K}\left(p_{1}, p_{1}-2\right) \subset Q_{K}\left(p_{2}, p_{2}-2\right)$ for $0<p_{1} \leq p_{2}<\infty$. Hence Corollary 6 implies that $Q_{K}(p, p-2)$ does not contain any singular inner functions. Now, since each inner function can be presented as a product of a Blaschke product and a singular inner function, the statement (ii) follows by Corollary 4 .

Remark 8. In many cases, $Q_{K}(p, p-2)$ contains also nontrivial Blaschke products unlike $Q_{K}(p, p-2-\varepsilon)$ for $\varepsilon \in(0, p)$. The contrast between these spaces is strong also in the general case. More precisely, using the inclusion $Q_{K}(p, q) \subset \mathscr{B}^{(q+2) / p}$ and [1, Theorem 5.1], we find that if $f \in Q_{K}(p, p-2-\varepsilon)$, then $f\left(e^{i \theta}\right) \in \Lambda_{\varepsilon / p}$; that is, the boundary function satisfies the Lipschitz condition of order $\varepsilon / p$ [1]. In particular, $f$ belongs to the disc algebra $\mathscr{A}$. On the other hand, by $[10$, Corollary 3.1], it is easy to find $K$ such that $\log (1-z)$ belongs to $Q_{K}(2,0)$.

Next we prove an alternative version of Theorem 1 . The proof uses some ideas from $[17,18]$. For the result, denote $\widehat{p}=$ $\min \{p, 1\}$.

Theorem 9. Let $p \in(1 / 2, \infty)$, and assume that $K$ satisfies the following conditions:

(i) $K$ is nondecreasing.

(ii) $\int_{0}^{1}(1-r)^{p-2} K(\log (1 / r)) r d r<\infty$.

(iii) $\int_{0}^{1} \varphi_{K}(r) r^{\widehat{p}-2} d r<\infty$.

(iv) $\int_{1}^{\infty} \varphi_{K}(r) r^{-\hat{p}^{-1}} d r<\infty$.

Then an inner function belongs to $Q_{K}(p, p-2)$ if and only if it is a Blaschke product associated with a sequence $\left\{z_{n}\right\}$ satisfying (4).

Proof. By a similar manner as in the proof of Proposition 7 , we may assume that $K$ satisfies the basic assumption of [11], which means that, in addition to (i) and (ii), $K$ is continuous, $K(r) \asymp K(2 r)$ for $r \in(0, \infty)$ and $K(s)=K(1)$ for $s \in[1, \infty)$.

Assume first that an inner function $B$ belongs to $Q_{K}(p, p-$ $2)$ for some $p \in(1 / 2, \infty)$. Then Proposition 7 implies that $B$ is a Blaschke product. Hence, using [11, Theorem 10], we obtain that the zero sequence of $B$ satisfies (4). 
If $B$ is a Blaschke product associated with a sequence $\left\{z_{n}\right\}$ satisfying (4), then [11, Lemma 5] with parameters $t=\widehat{p}-2$ and $c=2(\widehat{p}-1)$ yields

$$
\begin{aligned}
& \sup _{a \in \mathbb{D}} \sum_{n}\left(1-\left|\varphi_{a}\left(z_{n}\right)\right|\right)^{\hat{p}} K(1-|z|) \\
& \cdot \int_{\mathbb{D}} \frac{K}{\left|1-\overline{\varphi_{a}\left(z_{n}\right) z}\right|^{2 \widehat{p}}(1-|z|)^{2-\hat{p}}} d A(z)<\infty .
\end{aligned}
$$

Therefore $B \in Q_{K}(p, p-2)$ by [11, Proposition 8], and hence the assertion follows.

We proceed to prove Theorem 1 . First, it is known that $K$ satisfies (i) of Theorem 9. On the other hand, since $\log (1 / r) \asymp$ $1-r$ for $r \in(1 / e, 1)$, Lemma $\mathrm{A}$ (ii) together with the assumption yields

$$
\begin{aligned}
& \int_{0}^{1}(1-r)^{p-2} K\left(\log \frac{1}{r}\right) r d r \\
& \approx K(1) \\
& \quad+\int_{1 / e}^{1}(1-r)^{p-2} \frac{K(\log (1 / r))}{(\log (1 / r))^{\beta}}\left(\log \frac{1}{r}\right)^{\beta} d r \\
& \leq K(1) \int_{1 / e}^{1}(1-r)^{\beta+p-2} d r<\infty .
\end{aligned}
$$

Therefore the condition (ii) holds. Moreover, we obtain

$$
\begin{aligned}
\int_{0}^{1} \varphi_{K}(r) r^{\widehat{p}-2} d r & =\int_{0}^{1} \sup _{t \in(0,1)} \frac{K(r t)(r t)^{\beta}}{K(t)(r t)^{\beta}} r^{\widehat{p}-2} d r \\
& \lesssim \int_{0}^{1} r^{\beta+\widehat{p}^{-2}} d r<\infty
\end{aligned}
$$

and consequently (iii) is satisfied. Regarding (iv), we may assume, by Lemma $\mathrm{A}(\mathrm{ii})$, that there exists $\alpha \in[\beta, \widehat{p})$ such that $r^{-\alpha} K(r) \searrow$ for all $r \in(0, \infty)$. Thus

$$
\begin{aligned}
\int_{1}^{\infty} \varphi_{K}(r) r^{-\widehat{p}^{-1}} d r & =\int_{1}^{\infty} \sup _{t \in(0,1)} \frac{K(r t)(r t)^{\alpha}}{K(t)(r t)^{\alpha}} r^{-\widehat{p}-1} d r \\
& \lesssim \int_{1}^{\infty} r^{\alpha-\widehat{p}^{-1}} d r<\infty ;
\end{aligned}
$$

and hence, the assertion finally follows by Theorem 9 .

We end this paper with the following remark.

Remark 10. We make the following observations about Theorem 1 and Proposition 7:

(i) If $K(r)=r^{s}$ with $s \in[0, \infty)$, then $Q_{K}(p, q)=$ $F(p, q, s)[19,20]$. Related to $F(p, q, s)$, Theorem 1 and Proposition 7 generalize [9, Theorems 1.3 and 1.4]. Moreover, by [10, Lemmas 2.1, 2.2 and Corollary 3.1], it is easy to see that Theorem 1 generalizes the essential content of [10, Theorem 5.1]. (ii) The Möbius invariance of $Q_{K}(p, p-2)$ plays an important role in the proof of [11, Theorem 10]. Hence, if one want to characterize Blaschke products in $Q_{K}(p, q)$-spaces where $p<q+2$, using a similar technique as in this paper, it is necessary to assume that $Q_{K}(p, q)$ is Möbius invariant.

\section{Conflict of Interests}

The author declares that there is no conflict of interests regarding the publication of this paper.

\section{Acknowledgments}

The research reported in this paper was supported in part by the Väisälä Foundation, the Academy of Finland, Project no. 268009, and the Faculty of Science and Forestry of the University of Eastern Finland, Project no. 930349. The author thanks the University of Malaga for hospitality during his visit there.

\section{References}

[1] P. Duren, Theory of $\mathrm{H}^{p}$ Spaces, Academic Press, New York, NY, USA, 1970.

[2] P. Colwell, Blaschke Products. Bounded Analytic Functions, University of Michigan Press, Ann Arbor, Mich, USA, 1985.

[3] J. Mashreghi, Derivatives of Inner Functions, vol. 31 of Fields Institute Monographs, Springer, New York, NY, USA, 2013.

[4] P. R. Ahern, "The Poisson integral of a singular measure," Canadian Journal of Mathematics, vol. 35, no. 4, pp. 735-749, 1983.

[5] P. R. Ahern and D. N. Clark, "On inner functions with $B^{p}$ derivative," The Michigan Mathematical Journal, vol. 23, no. 2, pp. 107-118, 1976.

[6] P. R. Ahern and D. N. Clark, "On inner functions with $H^{p_{-}}$ derivative," Michigan Mathematical Journal, vol. 21, no. 2, pp. $115-127,1974$.

[7] H. Wulan and J. Zhou, "Q $Q_{K}$ type spaces of analytic functions," Journal of Function Spaces and Applications, vol. 4, no. 1, pp. 73$84,2006$.

[8] H. Wulan and J. Zhou, "The higher order derivatives of $Q_{K}$ type spaces," Journal of Mathematical Analysis and Applications, vol. 332, no. 2, pp. 1216-1228, 2007.

[9] F. Pérez-González and J. Rättyä, "Inner functions in the Möbius invariant Besov-type spaces," Proceedings of the Edinburgh Mathematical Society, vol. 52, pp. 751-770, 2009.

[10] M. Essén, H. Wulan, and J. Xiao, "Several function-theoretic characterizations of Möbius invariant $Q_{K}$ spaces," Journal of Functional Analysis, vol. 230, pp. 78-115, 2006.

[11] C. Yang, "Inner functions in $Q_{k}$ type spaces," Journal of Function Spaces and Applications, vol. 9, no. 3, pp. 305-322, 2011.

[12] A. L. Shields and D. L. Williams, "Bounded projections, duality, and multipliers in spaces of analytic functions," Transactions of the American Mathematical Society, vol. 162, pp. 287-302, 1971.

[13] B. Muckenhoupt, "Hardy's inequality with weights," Studia Mathematica, vol. 44, no. 1, pp. 31-38, 1972.

[14] W. Rudin, Real and Complex Analysis, McGraw-Hill, New York, NY, USA, 1987. 
[15] D. A. Stegenga, "Bounded Toeplitz operators on $H^{1}$ and applications of the duality between $H^{1}$ and the functions of bounded mean oscillation," American Journal of Mathematics, vol. 98, no. 3, pp. 573-589, 1976.

[16] H. Wulan and K. Zhu, "Lacunary series in $Q_{K}$ spaces," Studia Mathematica, vol. 178, no. 3, pp. 217-230, 2007.

[17] M. Essén and J. Xiao, "Some results on $Q_{p}$ spaces, $0<p<1$," Journal für die Reine und Angewandte Mathematik, vol. 485, pp. 173-195, 1997.

[18] I. È. Verbitskiî, "Multipliers in spaces with 'fractional' norms, and inner functions," Sibirskii Matematicheskii Zhurnal, vol. 26, pp. 51-72, 1985.

[19] J. Rättyä, "On some complex function spaces and classes," Annales Academice Scientiarum Fennica. Mathematica Dissertationes, vol. 124, pp. 1-73, 2001.

[20] R. Zhao, "On a general family of function spaces," Annales Academice Scientiarum Fennice. Mathematica Dissertationes, vol. 105, pp. 1-56, 1996. 


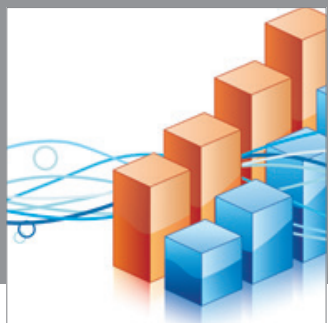

Advances in

Operations Research

mansans

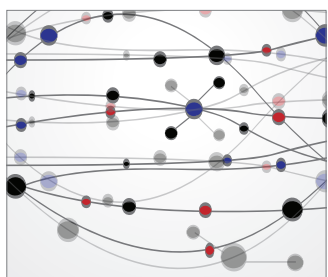

The Scientific World Journal
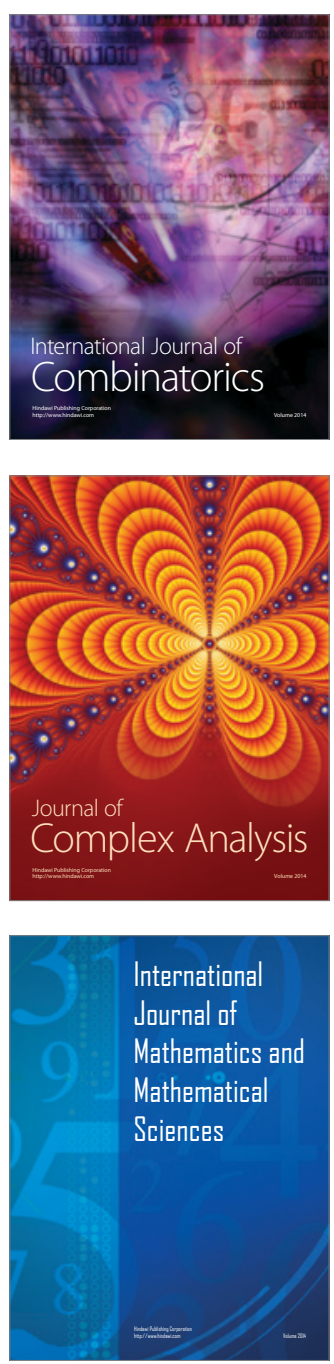
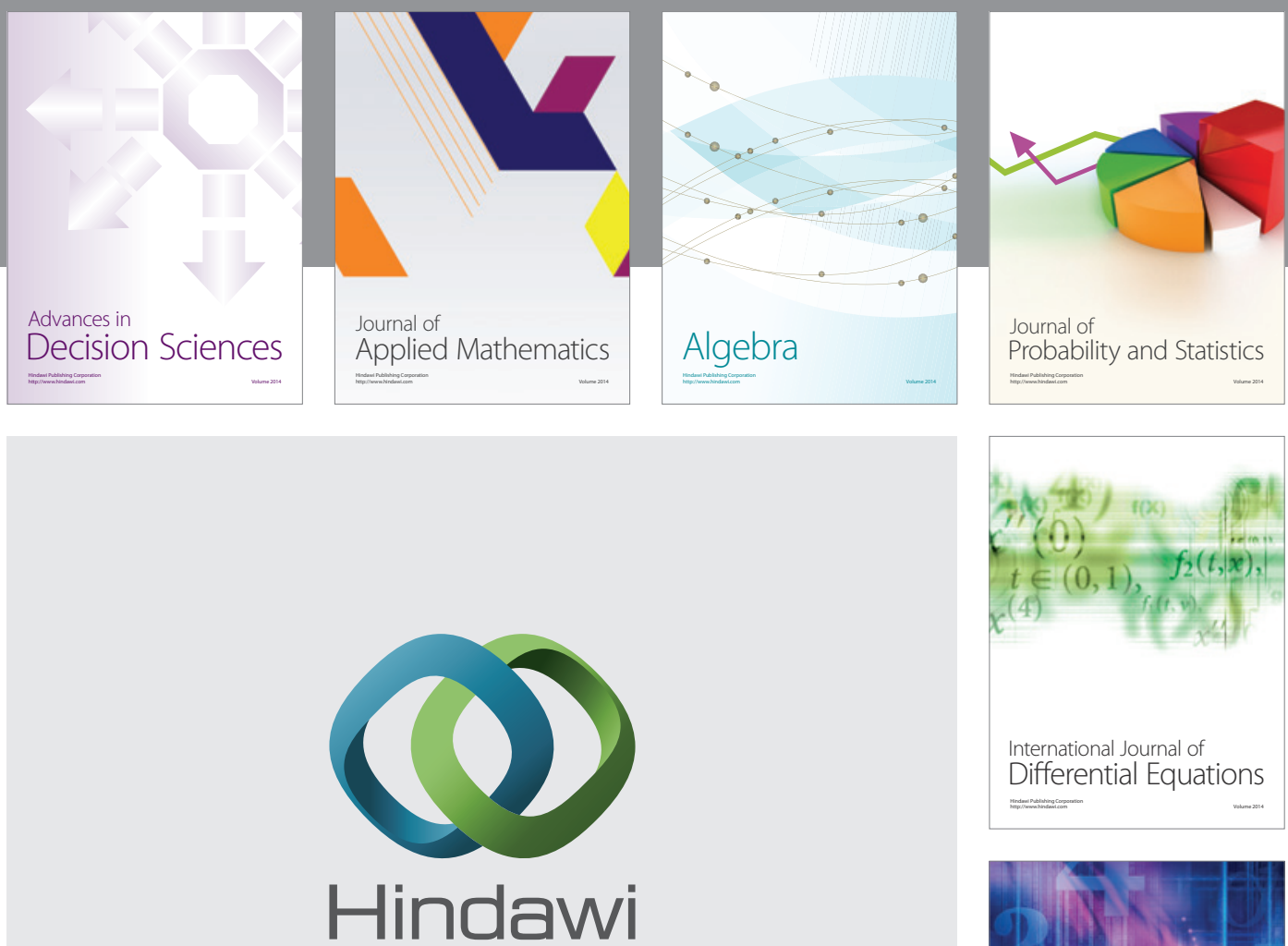

Submit your manuscripts at http://www.hindawi.com
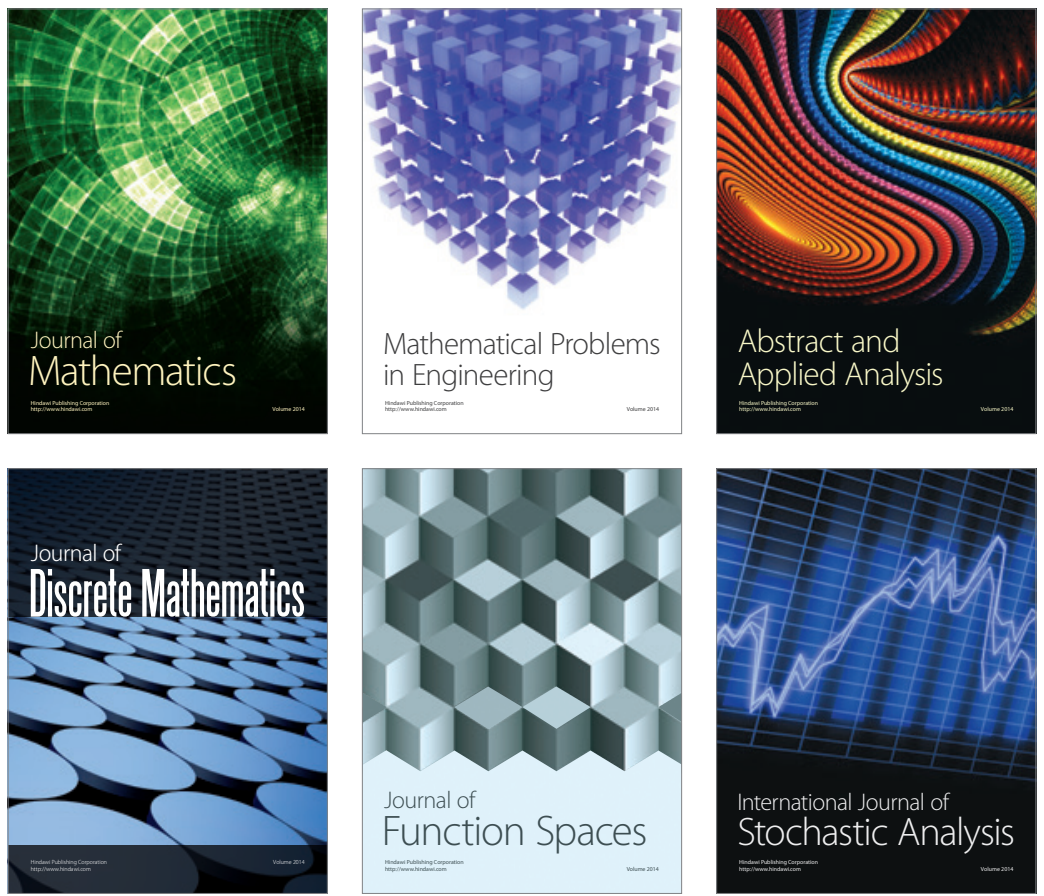

Journal of

Function Spaces

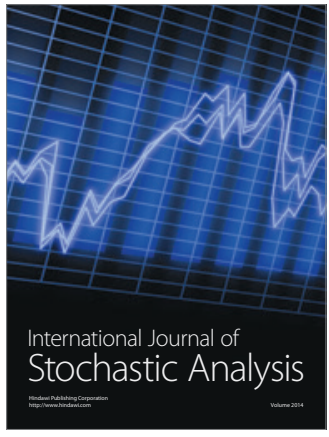

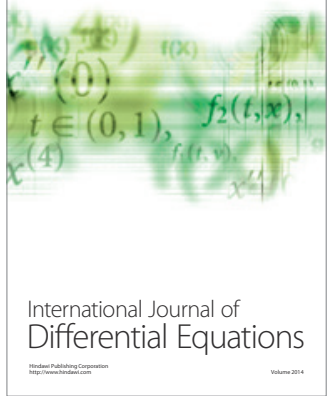
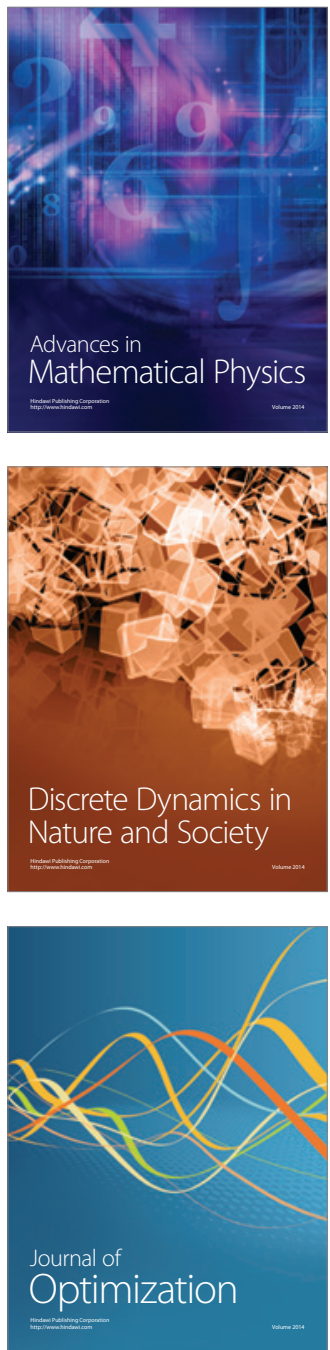